\title{
BMJ Open Pattern of cardiac surveillance among patients with lymphoma receiving anthracycline-based chemotherapy
}

\author{
Olivia Y Hung, ${ }^{1}$ Jennifer R Brown, ${ }^{1}$ Tian Dai, ${ }^{2}$ Kirk A Easley, ${ }^{2}$ \\ Christopher R Flowers, ${ }^{3}$ Susmita Parashar $^{1}$
}

To cite: Hung OY, Brown JR, Dai T, et al. Pattern of cardiac surveillance among patients with lymphoma receiving anthracycline-based chemotherapy. BMJ Open 2015;5:e008350. doi:10.1136/bmjopen-2015008350

- Prepublication history and additional material is available. To view please visit the journal (http://dx.doi.org/ 10.1136/bmjopen-2015008350).

Received 30 March 2015 Revised 1 July 2015 Accepted 24 July 2015

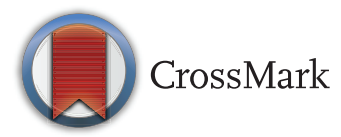

${ }^{1}$ Division of Cardiology, Department of Medicine, Emory University School of Medicine, Atlanta, Georgia, USA

${ }^{2}$ Department of Biostatistics \& Bioinformatics, Rollins School of Public Health, Emory University, Atlanta, Georgia, USA

${ }^{3}$ Division of Hematology \& Medical Oncology, Department of Medicine, Emory University School of Medicine, Atlanta, Georgia, USA

Correspondence to Dr Susmita Parashar; susmita.parashar@emory.edu

\section{ABSTRACT}

Objective: Anthracyclines are potent antineoplastic agents in the treatment of lymphoid malignancies, but their therapeutic benefit is limited by cardiotoxicity. The American Heart Association (AHA) recommends routine surveillance, early diagnosis and treatment of anthracycline-based chemotherapy $(\mathrm{AC})$ induced cardiomyopathy (AC-CMP). We aimed to assess the prevalence of AC-CMP in patients with lymphoma, surveillance patterns of left ventricular ejection fraction (LVEF) in those receiving $A C$ and management of patients with AC-CMP at an academic medical centre prior to the development of a comprehensive cardiooncology programme.

Methods: We performed a retrospective cohort study examining 218 patients with aggressive B cell nonHodgkin's lymphomas (B-NHL) who received AC 1992-2012 and had serial follow-up. AC-CMP was defined as LVEF decrease $\geq 10 \%$ with final LVEF $\leq 50 \%$ or LVEF reduction $\geq 15 \%$ regardless of final LVEF. Results: Of 218 patients treated with AC, 73 (34\%) had LVEF assessment both prior to and after receiving AC. Of these 73 patients, 24 developed AC-CMP and had higher cumulative all-cause mortality than those without AC-CMP (HR 2.35, $\mathrm{p}=0.03$ ). Coronary artery disease (CAD) was an independent predictor of $A C$ CMP ( $p=0.048)$. Mean post-AC LVEF was lower in patients with CAD compared with those without CAD when their baseline LVEF was $45 \%(p=0.0009)$ or $55 \%$ $(p=0.001)$ but was similar at $65 \%(p=0.33)$. Less than half of patients with AC-CMP received recommended heart failure medication therapy.

Conclusions: Historically, one-third of patients with B-NHL treated with AC underwent surveillance according to AHA guidelines. There is substantial opportunity for collaboration between oncologists and cardiologists to improve the care of patients with lymphoma receiving $\mathrm{AC}$.

\section{INTRODUCTION}

The American Cancer Society estimates that in 2014, there were approximately 14.5 million children and adults with a history of cancer including 297820 male and 272000 female survivors of non-Hodgkin's lymphoma

\section{Strengths and limitations of this study}

- Comprehensive single institution approach of using evidence-based guidelines to evaluate realworld surveillance and management patterns of patients with lymphoma with anthracycline-based chemotherapy-induced cardiomyopathy.

- Retrospective study of 218 patients at a single institution with information on demographics, medical comorbidities, left ventricular ejection fraction and survival data.

- Generalisability of the results is limited to a subset of the targeted population that received the expected surveillance and treatment according to the American Heart Association guidelines.

(NHL). ${ }^{1}$ While the development of effective diagnostic and chemotherapeutic strategies has resulted in a large population of long-term cancer survivors, the impact of chemotherapy on the long-term health of these survivors is substantial. Cardiac toxicity is a common complication of anthracycline-based chemotherapy (AC), with the clinical course ranging from transient asymptomatic left ventricular dysfunction (LVD) to chronic heart failure (HF) and even cardiac death. The most common clinical presentation of AC cardiotoxicity is a dose-dependent cardiomyopathy (CMP) that leads to $\mathrm{HF}^{2-5}$ Symptomatic HF is the most serious form of AC-CMP, with an incidence of $5-50 \%$, depending on the cumulative dose of AC received. ${ }^{6-9}$ Asymptomatic CMP manifested by echocardiographic abnormalities is more common than symptomatic disease and, depending on the definitions applied, can be found in approximately $50 \%$ of all patients who received AC. ${ }^{8}$

Compared with other more frequent forms of CMP, AC-CMP has been associated with an especially poor prognosis, with a 2-year mortality rate up to $60 \% .^{10}$ Importantly, AC-CMP also limits the therapeutic choices for 
patients with relapsed cancer to less intensive and potentially less effective cancer therapies. ${ }^{2}$ However, recent studies have indicated that in AC-CMP, left ventricular ejection fraction (LVEF) recovery and adverse cardiac event reduction may be achieved when asymptomatic cardiac dysfunction is detected early and modern $\mathrm{HF}$ therapy is promptly initiated. ${ }^{3} 11$ Therefore, the American College of Cardiology/American Heart Association/American Society of Echocardiography (ACC/AHA/ASE) committee gives a class I recommendation for regular LVEF monitoring in patients exposed to cardiotoxic agents in order to facilitate decisions regarding further chemotherapy as well as provide early diagnosis and treatment of AC-CMP. ${ }^{12-14}$ Echocardiographic surveillance is also supported by the European Society for Medical Oncology (ESMO). ${ }^{15}$

Despite these recommendations, there are limited data regarding surveillance patterns and cardiovascular treatment for patients with lymphoma receiving AC. NHLs are characterised by abnormal tissue growth in the lymphatic system and is the sixth most common cancer in both men and women, with increasing incidence among all age groups. ${ }^{16}$ Aggressive subtypes of B cell NHLs (B-NHLs) such as diffuse large B cell lymphoma and Burkitt lymphoma are commonly cured with anthracycline chemoimmunotherapy while other subtypes such as follicular lymphoma and mantle cell lymphoma often require systemic AC. ${ }^{17}$ We therefore conducted a retrospective cohort study of patients with aggressive B-NHLs who were treated with AC to assess the cardiac surveillance and HF management of these patients at an academic medical centre prior to the development of a comprehensive cardio-oncology programme and to examine clinical characteristics that may impact AC-CMP development or mortality.

\section{METHODS}

\section{Participants and data collection}

We used the Emory University Lymphoma Enterprise Architecture Data-system (LEAD), a database that integrates genetic, clinical, histological, epidemiological and clinical trial information from disparate medical systems for improved research, disease diagnosis, treatment and statistical outcomes measurements. The process for managing the LEAD registry and incorporating new patient data into LEAD is approved by the Emory University Institutional Review Board (IRB) and data collection and extraction procedures have previously been described. ${ }^{18} 19$ Patients with NHL diagnoses who had received $\mathrm{AC}$ were identified through a series of search strategies including International Classification of Disease-Oncology (ICD-O) histology codes and freetext searches of anatomical pathology reports and electronic medical records. An IRB waiver of consent was obtained for the data analysis required for this current study. The retrospective cohort for the current analysis was limited to patients with aggressive B-NHLs who received $\mathrm{AC}$ and evaluation during their care at an Emory Healthcare facility.

\section{Predictor variables}

Comprehensive chart review was undertaken to identify patients who underwent LVEF evaluation both prior to and after AC and to collect sociodemographic data (eg, age, sex, race), comorbid conditions (eg, hypertension, hyperlipidaemia, diabetes mellitus, coronary artery disease $(\mathrm{CAD}))$, health status and treatment variables (eg, mediastinal radiation, method of LVEF assessment). All available imaging data were reviewed by an independent researcher (JRB). Any disagreements between the reader and the original report (difference in LVEF $>5$ absolute points) was solved by consensus reached with subsequent joint evaluation (JRB and SP). Independent reports have suggested two-dimensional transthoracic echocardiography (TTE) test-retest variability of around 5-7\%. ${ }^{20}$

\section{Outcome variables}

The primary outcome measures were (1) documented pre-AC and post-AC LVEF evaluations and (2) development of AC-CMP, among patients with B-NHL treated with AC. Consistent with current guidelines, AC-CMP was defined as a LVEF reduction by $\geq 10 \%$ concomitant with an absolute post-AC LVEF $\leq 50 \%$, or a LVEF reduction of $\geq 15 \%$ regardless of post-AC LVEF. ${ }^{22}$

Secondary outcome measures included vital status after the first post-AC LVEF evaluation as well as AC-CMP management. Mortality data were obtained using chart documentation and the Social Security Death Index. For three patients referred to hospice but without a verified exact date of death, the last recorded date of patient contact in the electronic medical record was used as the date of death. Follow-up time period was defined from the patient's first $\mathrm{AC}$ at our institution to the date of their last known clinic visit or date of death. CMP management was defined using standard guideline recommendations for patients with decreased $\mathrm{LVEF}^{23}$ such as treatment with ACE inhibitors, $\beta$-blocker therapy, serial imaging and/or cardiology referral.

\section{Statistical analysis}

The prevalence of CMP was estimated as a single sample proportion. A 95\% CI based on Wilson's score method for a single sample proportion was calculated for the prevalence of CMP. ORs were calculated to measure the degree of association between risk factors and AC-CMP. Only risk factors that were significant at $\mathrm{p}<0.20$ in the univariable analyses were included in the multivariable analyses (Cox proportional hazards regression analysis). A $p$ value of 0.20 was used as a significance level to help ensure that all potentially important covariates were included in the final multivariable Cox model. The HR and its 95\% CI were calculated for each factor in the presence of others in the final Cox proportional hazards regression model. 
Using linear regression, post-LVEF was regressed on initial LVEF and compared in patients with and without specific risk factors for AC-CMP. History of CAD was the only independent predictor identified, so this factor and the interaction between initial LVEF and CAD history were included as covariates. Adjusted post-LVEF means were estimated for patients with and without a history of $\mathrm{CAD}$ and defined as the predicted response values obtained by evaluating the regression equation for CAD at the mean initial LVEF. Results are provided as adjusted means with $95 \%$ CIs.

Cumulative survival was estimated with the Kaplan-Meier method. Log-rank tests were used to compare survival according to demographic and clinical characteristics. Age of the patient was evaluated as a categorical covariates (above or below the median age of 60 , or per 10-year increase) and as a continuous covariate. Only risk factors that were significant at $p<0.20$ in univariable analyses were included in the multivariable analyses. The HR and its $95 \%$ CI were calculated for each factor in the presence of others in the final Cox proportional hazards regression model.

\section{RESULTS}

Of 506 patients in the LEAD registry who received AC for aggressive B-NHL between 1995 and 2012, 218 patients completed $\mathrm{AC}$ and regular follow-up within the Emory Healthcare system. In total, 73 of the 218 patients (34\%) received imaging surveillance of LVEF with either TTE or multigated acquisition scan (MUGA) both prior to and after receiving chemotherapy, whereas 67 patients $(30 \%)$ had imaging surveillance only prior to receiving chemotherapy, 9 patients $(4 \%)$ received imaging surveillance only after receiving chemotherapy and $69(32 \%)$ had no documentation of imaging surveillance within the electronic medical record (figure 1). Of the 73 patients who underwent both baseline and follow-up LVEF examinations, 13 patients underwent MUGA, 59 patients underwent echocardiography and 1 patient underwent positron emission tomography prior to receiving AC. The same modality was used for serial LVEF examinations in $93 \%$ of patients. Follow-up diastolic function was evaluated in 27 patients.

The prevalence of AC-CMP was estimated at 33\% (95\% CI $23 \%$ to $44 \%$ ). Eleven of the 24 patients who developed AC-CMP had mildly reduced LVEF (post-AC $\mathrm{LVEF}>45 \%)$ and the remaining 13 had moderately to severely reduced LVEF (post-AC $\mathrm{LVEF} \leq 45 \%$ ). Among patients receiving $\mathrm{AC}$, patients with $\mathrm{CAD}$ were more likely to develop AC-CMP (table 1). As CAD history was the only significant independent predictor $(p=0.048)$, adjusted post-AC LVEF means were compared in patients with and without CAD using linear regression (table 2).

Post-AC LVEF in these two groups differed significantly depending on the baseline LVEF ( $p=0.01$, test for interaction between baseline LVEF and CAD). Mean post-AC LVEF was significantly lower in patients with CAD compared with patients without CAD when baseline LVEF was $45 \%$ (36\% vs $58 \%, \mathrm{p}=0.0009)$ and when baseline LVEF was $55 \%$ ( $43 \%$ vs $56 \%, \mathrm{p}=0.001$ ). However, mean post-AC LVEF was similar in patients with CAD compared with patients without $\mathrm{CAD}$ when their baseline LVEF was $65 \%$ ( $50 \%$ vs $53 \%$, $\mathrm{p}=0.33$ ).

Median follow-up was 2.99 years. Cumulative all-cause mortality rates were higher in patients who developed AC-CMP compared with those who did not (figure 2 and table 3). This difference remained significant $(\mathrm{p}=0.03)$ even after adjusting for age, sex, hyperlipidaemia and

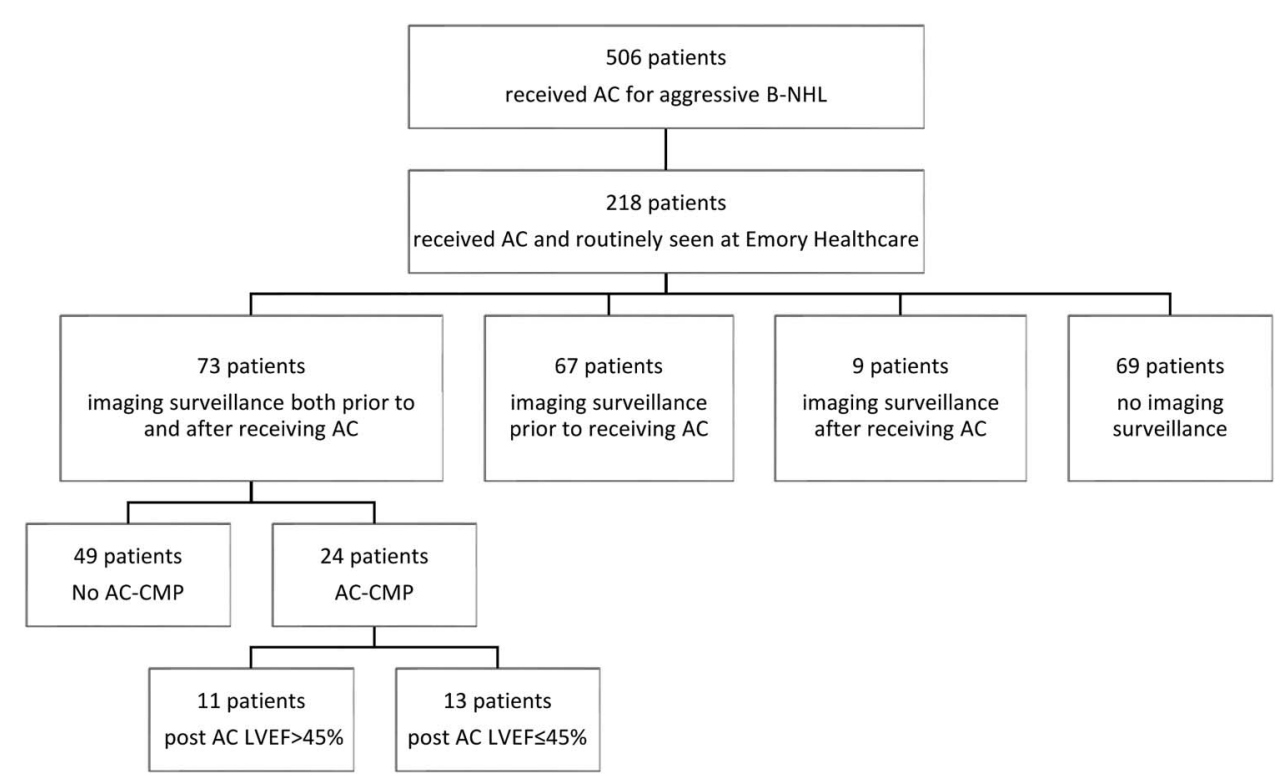

Figure 1 Surveillance pattern among patients with aggressive non-Hodgkin's lymphoma who received anthracycline-based chemotherapy. Imaging surveillance of LVEF was performed with either transthoracic echocardiogram or multigated acquisition scan (AC, anthracycline-based chemotherapy; AC-CMP, anthracycline-based chemotherapy-induced cardiomyopathy; B-NHL, B cell non-Hodgkin's lymphoma; LVEF, left ventricular ejection fraction). 
history of CAD (table 4). Interestingly, hyperlipidaemia was an independent prognostic factor for survival after adjusting for age, sex and CAD history.

Of the 24 patients with AC-CMP, 11 were referred to cardiology, 7 received $\beta$-blocker therapy, 8 received treatment with ACE inhibitors and 8 underwent additional LVEF evaluation. Six patients were prescribed both $\beta$-blocker and ACE inhibitor therapy and only three patients received $\beta$-blocker and ACE inhibitor therapy in addition to repeat TTE (figure 3 ).

\section{DISCUSSION}

The benefits of AC are limited by cumulative dose-related cardiotoxicity. ${ }^{24}$ In this study, we observed that (1) 73 patients (34\%) who completed AC at a single academic medical centre for aggressive B-NHL had imaging surveillance both prior to and after receiving chemotherapy, (2) $33 \%$ of patients who underwent LVEF evaluation both prior to and after receiving AC developed CMP, (3) patients with CAD were more likely to develop AC-CMP and this was influenced by their baseline LVEF and (4) a minority of patients who developed AC-CMP were referred to a cardiologist, started on HF medications such as $\beta$-blockers or ACE inhibitors, or had repeat imaging for further LVEF evaluation.
Table 2 Adjusted post-AC mean LVEF in patients with lymphoma with and without $C A D$

\begin{tabular}{llll}
\hline \multicolumn{3}{c}{$\begin{array}{l}\text { Adjusted } \\
\text { post-LVEF }\end{array}$} \\
CAD & Pre-LVEF (\%) & $\begin{array}{l}\text { mean (\%) } \\
\text { Yes }(\mathrm{n}=12)\end{array}$ & All, mean=59 Cl \\
& 45 & 47 & $(41$ to 53) \\
& 55 & 36 & $(27$ to 45$)$ \\
& 65 & 43 & $(37$ to 49$)$ \\
No $(\mathrm{n}=61)$ & All, mean=62 & 50 & $(43$ to 56$)$ \\
& 45 & 58 & $(52$ to 57$)$ \\
& 55 & 56 & $(49$ to 67$)$ \\
& 65 & 53 & $(51$ to 60$)$ \\
& & $(50$ to 56$)$
\end{tabular}

The median years on study for survivors were 2.99 years. $\mathrm{AC}$, anthracycline-based chemotherapy; CAD, coronary artery disease; LVEF, left ventricular ejection fraction.

Anthracycline-induced cardiotoxicity ranges from asymptomatic mild LVD to severe HF that decreases patients' quality of life and life expectancy. In our investigation, we observed that patients with AC-CMP were 2.36 times more likely to die than those without AC-CMP. Furthermore, patients who had CAD and LVD on their baseline LVEF assessment were more likely to develop further reduced LVEF and AC-CMP, thereby

Table 1 Univariable analysis of clinical characteristics potentially associated with AC-CMP among 73 patients with lymphoma with left ventricular ejection fraction assessment before and after receiving anthracycline-based chemotherapy

\begin{tabular}{|c|c|c|c|c|c|}
\hline Characteristic & Total $(n=73)$ & AC-CMP $(n=24)$ & No AC-CMP $(n=49)$ & OR (95\% Cl) & p Value \\
\hline \multicolumn{4}{|c|}{ Mediastinal radiation } & $1.26(0.27$ to 5.76$)$ & 0.77 \\
\hline Yes & 8 & 3 & 5 & & \\
\hline No & 65 & 21 & 44 & & \\
\hline Age & 73 & $59.0 \pm 15.5$ & $60.0 \pm 13.2$ & $1.00(0.96$ to 1.03$)$ & 0.82 \\
\hline \multicolumn{4}{|l|}{ Sex } & $0.74(0.27$ to 2.00$)$ & 0.55 \\
\hline Female & 31 & 9 & 22 & & \\
\hline Male & 42 & 15 & 27 & & \\
\hline \multicolumn{4}{|l|}{ Race } & 0.91 (0.28 to 3.02$)$ & 0.88 \\
\hline Black & 17 & 5 & 12 & & \\
\hline Caucasian & 51 & 16 & 35 & & \\
\hline Unknown & 5 & 3 & 2 & & \\
\hline \multicolumn{4}{|c|}{ Smoking history } & $1.21(0.38$ to 3.85$)$ & 0.75 \\
\hline Yes & 19 & 6 & 13 & & \\
\hline No & 47 & 13 & 34 & & \\
\hline Unknown & 7 & 5 & 2 & & \\
\hline \multicolumn{4}{|l|}{ Hypertension§ } & $1.34(0.50$ to 3.61$)$ & 0.56 \\
\hline Yes & 34 & 12 & 22 & & \\
\hline No & 38 & 11 & 27 & & \\
\hline \multicolumn{4}{|c|}{ Diabetes mellitus§ } & 1.85 (0.45 to 7.67$)$ & 0.39 \\
\hline Yes & 9 & 4 & 5 & & \\
\hline No & 63 & 19 & 44 & & \\
\hline \multicolumn{4}{|c|}{ Hyperlipidaemia§ } & 1.38 (0.43 to 4.40$)$ & 0.59 \\
\hline Yes & 16 & 6 & 10 & & \\
\hline No & 56 & 17 & 39 & & \\
\hline \multicolumn{4}{|c|}{ Coronary artery disease } & $3.62(1.01$ to 13.0$)$ & $0.048^{*}$ \\
\hline Yes & 12 & 7 & 5 & & \\
\hline No & 61 & 17 & 44 & & \\
\hline
\end{tabular}


Figure 2 Cumulative survival among 73 patients with lymphoma with left ventricular ejection fraction assessment before and after receiving anthracycline-based chemotherapy by the presence or absence of anthracycline-based chemotherapy-induced cardiomyopathy (CMP). Cumulative survival by presence or absence of CMP. The median years on study for survivors were 2.99 years.

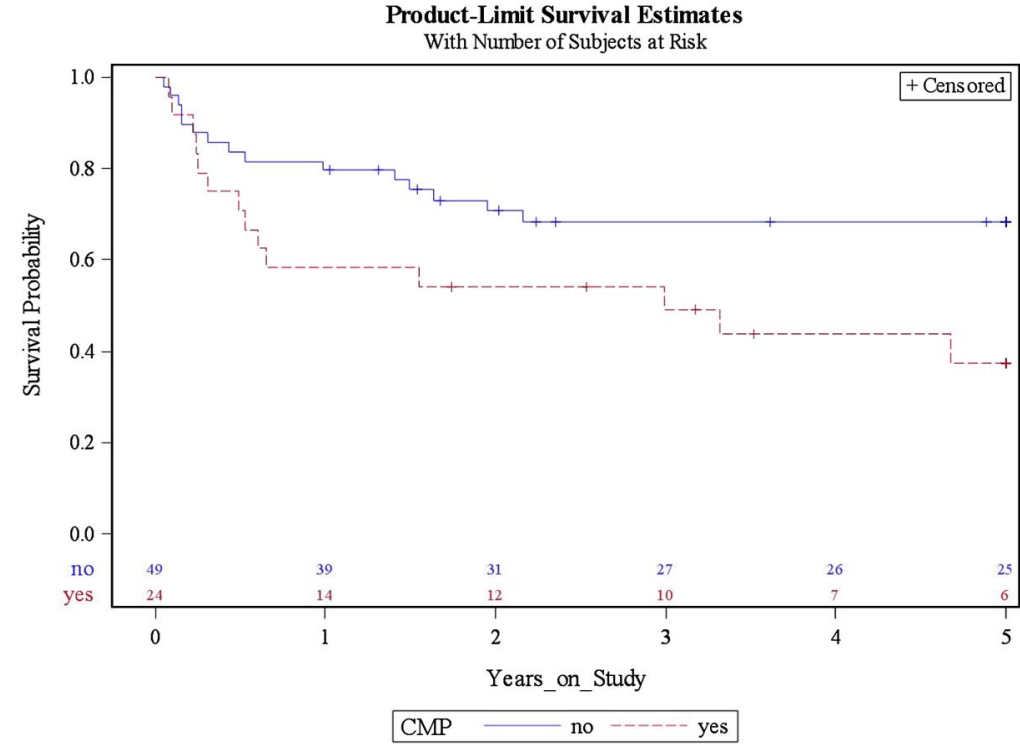

Table 3 Univariable analysis of clinical characteristics potentially associated with time to death among 73 patients with lymphoma with LVEF assessment before and after receiving anthracycline

\begin{tabular}{|c|c|c|c|c|c|}
\hline Characteristic & Total $(n=73)$ & Death $(n=34)$ & 1-Year survival (SE) & 5-Year survival (SE) & p Value \\
\hline \multicolumn{5}{|l|}{ AC-CMP } & \multirow[t]{3}{*}{$0.02^{*}$} \\
\hline Yes & 24 & 15 & $0.58(0.10)$ & $0.38(0.11)$ & \\
\hline No & 49 & 19 & $0.80(0.06)$ & $0.68(0.07)$ & \\
\hline \multicolumn{5}{|c|}{ Mediastinal radiation } & \multirow[t]{3}{*}{0.93} \\
\hline Yes & 8 & 4 & $0.88(0.12)$ & $0.58(0.19)$ & \\
\hline No & 65 & 30 & $0.71(0.06)$ & $0.59(0.06)$ & \\
\hline \multicolumn{5}{|l|}{ Age (years) } & \multirow[t]{3}{*}{$0.07^{*}$} \\
\hline$<60$ & 36 & 12 & $0.72(0.07)$ & $0.69(0.08)$ & \\
\hline$\geq 60$ & 37 & 22 & $0.73(0.07)$ & 0.49 (0.09) & \\
\hline \multicolumn{5}{|l|}{ Sex } & \multirow[t]{3}{*}{$0.15^{\star}$} \\
\hline Female & 31 & 11 & $0.77(0.08)$ & $0.63(0.09)$ & \\
\hline Male & 42 & 23 & $0.69(0.07)$ & $0.55(0.08)$ & \\
\hline \multicolumn{5}{|l|}{ Race } & \multirow[t]{4}{*}{0.57} \\
\hline Black & 17 & 7 & $0.82(0.09)$ & $0.70(0.11)$ & \\
\hline Caucasian & 51 & 25 & $0.71(0.06)$ & $0.55(0.07)$ & \\
\hline Unknown & 5 & 2 & & & \\
\hline \multicolumn{5}{|l|}{ Smoking history } & \multirow[t]{4}{*}{0.67} \\
\hline Yes & 19 & 8 & $0.84(0.08)$ & $0.71(0.11)$ & \\
\hline No & 47 & 20 & $0.72(0.07)$ & $0.61(0.07)$ & \\
\hline Unknown & 7 & 6 & & & \\
\hline \multicolumn{5}{|l|}{ Hypertension§ } & \multirow[t]{3}{*}{0.81} \\
\hline Yes & 34 & 17 & $0.76(0.07)$ & $0.61(0.09)$ & \\
\hline No & 38 & 16 & $0.71(0.07)$ & 0.58 (0.09) & \\
\hline \multicolumn{5}{|c|}{ Diabetes mellitus§ } & \multirow[t]{3}{*}{0.85} \\
\hline Yes & 9 & 4 & $0.78(0.14)$ & $0.56(0.17)$ & \\
\hline No & 63 & 29 & $0.73(0.06)$ & $0.60(0.06)$ & \\
\hline \multicolumn{5}{|c|}{ Hyperlipidaemia§ } & \multirow[t]{3}{*}{$0.11^{*}$} \\
\hline Yes & 16 & 4 & $0.88(0.08)$ & $0.73(0.12)$ & \\
\hline No & 56 & 29 & $0.70(0.06)$ & $0.55(0.07)$ & \\
\hline \multicolumn{5}{|c|}{ Coronary artery disease } & \multirow[t]{3}{*}{$0.16^{\star}$} \\
\hline Yes & 12 & 8 & $0.75(0.13)$ & $0.34(0.15)$ & \\
\hline No & 61 & 26 & $0.72(0.06)$ & $0.63(0.06)$ & \\
\hline
\end{tabular}


Table 4 Multivariable analysis of clinical characteristics potentially associated with time to death among 73 patients with lymphoma with LVEF assessment before and after receiving anthracycline

\begin{tabular}{|c|c|c|c|c|}
\hline Characteristic & & HR & $95 \% \mathrm{Cl}$ & p Value \\
\hline AC-CMP & Yes vs no & 2.36 & (1.11 to 5.02$)$ & 0.03 \\
\hline Sex & Female vs male & 0.68 & (0.32 to 1.42$)$ & 0.24 \\
\hline Hyperlipidaemia & Yes vs no & 0.27 & (0.09 to 0.86$)$ & 0.03 \\
\hline Coronary artery disease & Yes vs no & 1.79 & (0.68 to 4.77 ) & 0.24 \\
\hline Age & Per 10-year $\uparrow$ & 1.04 & (0.79 to 1.38$)$ & 0.78 \\
\hline
\end{tabular}

supporting the theory that if early LVD is not diagnosed or treated, additional AC may lead to irreversible HF. Many have proposed recommendations for early LVD detection in order to prevent transient LVD from becoming a debilitating complication of chemotherapy. For example, Schwartz et $a l^{25}$ reported that patients at high risk of developing HF could still be treated with AC provided that serial studies were performed after delivering set amounts of chemotherapy and that doxorubicin was discontinued when indicated. The AHA/ACC guidelines advise all patients undergoing treatment with anthracyclines to undergo baseline echocardiogram with assessment of both systolic and diastolic cardiac function and to monitor LVEF after administration of either half the planned dose or a cumulative dose of $300 \mathrm{mg} / \mathrm{m}^{2}$ of doxorubicin, as well as follow-up imaging at least 12 months after AC completion. ${ }^{13} 14$

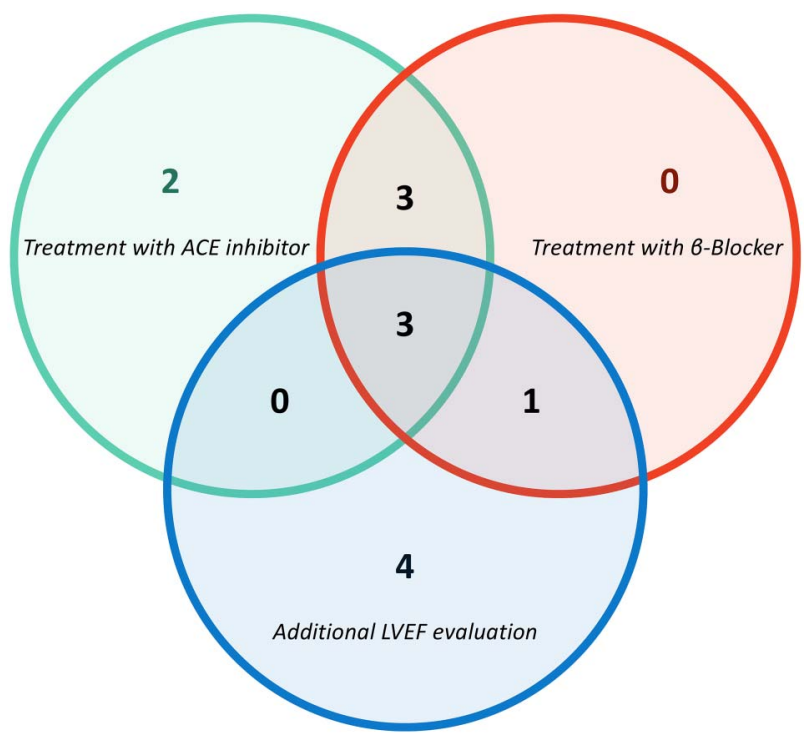

Figure 3 Venn diagram of patients with anthracycline-based chemotherapy-induced cardiomyopathy (AC-CMP) who received medication therapy and additional left ventricular ejection fraction (LVEF) surveillance. Thirteen of 24 patients with AC-CMP received medication therapy or underwent additional LVEF evaluation. Four patients only had additional LVEF evaluation and two patients were treated only with ACE inhibitors. Three patients received both ACE inhibitor and $\beta$-blocker medications while one patient received $\beta$-blocker therapy with repeat LVEF assessment. Three patients were treated with both ACE inhibitors and $\beta$-blockers and underwent additional LVEF evaluation.
This study found that only about one-third of patients who underwent AC for aggressive B-NHLs prior to the establishment of a comprehensive cardio-oncology programme had documentation of receiving the recommended LVEF assessments for AC-CMP monitoring. Cardinale $e a^{11}{ }^{26}$ demonstrated that, in the majority of patients, early detection and treatment of AC-CMP with standard of care HF therapy can result in partial or full recovery of LVEF. In our study, we observed that less than half of those who developed AC-CMP were referred to a cardiologist, received serial non-invasive cardiac monitoring or were treated with standard HF medications. These observations suggest that there is substantial opportunity for providers in oncology and cardiology to collaborate and improve on the current delivery of care in patients with AC-CMP.

This study does have several limitations as it is a small retrospective study at a single institution that focused on patients with B-NHL who received AC and regular follow-up. Patients who received care outside the Emory Healthcare system may have received the recommended surveillance and management, but their Emory records may be missing this information. The generalisability of our results is therefore limited to a subset of the targeted population that received the expected surveillance and treatment according to AHA guidelines. Nevertheless, to our knowledge, this is the first study to examine realworld surveillance and management practices of patients with AC-CMP. It is thus a comprehensive single institution approach to use evidence-based guidelines to evaluate practice patterns, which could eventually lead to practice improvement and provide metrics for other academic centres and community practices.

Barriers to improving AC-CMP surveillance patterns include unawareness of the scope of the problem, uncertainty regarding appropriate methods of diagnosis and management and lack of coordination between the two subspecialty fields. For example, the patients in this study were diagnosed with B-NHL during a period prior to the establishment of well-defined guidelines. The precise definition of cardiotoxicity and the recommendations for screening have not always been entirely clear; it has only been recently that both the Annals of Oncology and AHA/ ACC guidelines agreed on LVD monitoring and treatment of systolic HF with $\beta$-blockers and ACE inhibitors unless contraindicated. ${ }^{1415}$ Therefore, management of competing risks such as cancer relapse, treatment complications (eg, development of treatment-related cancers, infections, 
hypothyroidism, depression and/or atherosclerosis) and psychosocial factors have historically been prioritised.

In conclusion, this study showed that a minority of patients with aggressive B-NHL undergoing AC received guideline-based LVEF surveillance. Considering that reduced $\mathrm{LV}$ function is associated with worse morbidity and mortality, early identification of patients with LVD is essential to determine optimal oncological and cardiac management. Ideally, high-risk patients could be identified prior to developing LV dysfunction, and early identification and initiation of appropriate HF therapy could allow patients with mild cardiotoxicity to continue to receive $\mathrm{AC}$ under close observation and monitoring by a multidisciplinary team including both oncologists and cardiologists. $^{27} 28$ This study represents a first step towards overcoming barriers to effective AC-CMP management by documenting the scope of the problem and suggesting specific areas of improvement. Systematically tackling these challenges could lead to improved diagnosis and management of AC-CMP in patients with lymphoma.

Contributors OYH, JRB, CRF and SP were critically involved in the study design, collection and analysis as well as the preparation and editing of this manuscript. TD and KAE performed all statistical analyses in this study, provided substantial insight into data analysis and helped with manuscript preparation and editing.

Funding OYH is supported by the National Institutes of Health Ruth L. Kirschstein National Research Service Award training grant (5T32HL007745).

Competing interests None declared.

Ethics approval Emory University Institutional Review Board.

Provenance and peer review Not commissioned; externally peer reviewed.

Data sharing statement No additional data are available.

Open Access This is an Open Access article distributed in accordance with the Creative Commons Attribution Non Commercial (CC BY-NC 4.0) license, which permits others to distribute, remix, adapt, build upon this work noncommercially, and license their derivative works on different terms, provided the original work is properly cited and the use is non-commercial. See: http:// creativecommons.org/licenses/by-nc/4.0/

\section{REFERENCES}

1. American Cancer Society. Cancer treatment and survivorship facts \& figures 2014-2015. Atlanta: American Cancer Society, 2014.

2. Pai VB, Nahata MC. Cardiotoxicity of chemotherapeutic agents: incidence, treatment and prevention. Drug Saf 2000;22:263-302.

3. Herrmann J, Lerman A, Sandhu NP, et al. Evaluation and management of patients with heart disease and cancer: cardiooncology. Mayo Clin Proc 2014;89:1287-306.

4. Drafts BC, Twomley KM, D'Agostino R Jr, et al. Low to moderate dose anthracycline-based chemotherapy is associated with early noninvasive imaging evidence of subclinical cardiovascular disease. JACC Cardiovasc Imaging 2013;6:877-85.

5. Volkova M, Russell R III. Anthracycline cardiotoxicity: prevalence, pathogenesis and treatment. Curr Cardiol Rev 2011;7:214-20.

6. Cardinale D, Sandri MT, Colombo A et al. Prognostic value of troponin I in cardiac risk stratification of cancer patients undergoing high-dose chemotherapy. Circulation 2004;109:2749-54.

7. Pinder MC, Duan Z, Goodwin JS, et al. Congestive heart failure in older women treated with adjuvant anthracycline chemotherapy for breast cancer. J Clin Oncol 2007;25:3808-15.

8. Carver JR, Shapiro CL, $\mathrm{Ng} \mathrm{A}$, et al. American Society of Clinical Oncology clinical evidence review on the ongoing care of adult cancer survivors: cardiac and pulmonary late effects. J Clin Oncol 2007;25:3991-4008.

9. Carver JR, Szalda D, Ky B. Asymptomatic cardiac toxicity in long-term cancer survivors: defining the population and recommendations for surveillance. Semin Oncol 2013;40:229-38.
10. Felker GM, Thompson RE, Hare JM, et al. Underlying causes and long-term survival in patients with initially unexplained cardiomyopathy. N Engl J Med 2000;342:1077-84.

11. Cardinale D, Colombo A, Lamantia G, et al. Anthracycline-induced cardiomyopathy: clinical relevance and response to pharmacologic therapy. J Am Coll Cardiol 2010;55:213-20.

12. Cheitlin MD, Armstrong WF, Aurigemma GP, et al., American College of Cardiology; American Heart Association; American Society of Echocardiography. ACC/AHA/ASE 2003 guideline update for the clinical application of echocardiography: summary article: a report of the American College of Cardiology/American Heart Association Task Force on Practice Guidelines (ACC/AHA/ASE Committee to update the 1997 guidelines for the clinical application of echocardiography). Circulation 2003;108:1146-62.

13. Yancy CW, Jessup M, Bozkurt B, et al., American College of Cardiology Foundation/American Heart Association Task Force on Practice Guidelines. 2013 ACCF/AHA guideline for the management of heart failure: a report of the American College of Cardiology Foundation/American Heart Association Task Force on practice guidelines. Circulation 2013;128:e240-327.

14. Hunt SA, Abraham WT, Chin MH, et al. 2009 focused update incorporated into the ACC/AHA 2005 guidelines for the diagnosis and management of heart failure in adults: a report of the American College of Cardiology Foundation/American Heart Association Task Force on Practice Guidelines: developed in collaboration with the International Society for Heart and Lung Transplantation. Circulation 2009;119:e391-479.

15. Bovelli D, Plataniotis G, Roila F, et al., ESMO Guidelines Working

Group. Cardiotoxicity of chemotherapeutic agents and radiotherapy-related heart disease: ESMO Clinical Practice Guidelines. Ann Oncol 2010;21(Suppl 5):v277-82

16. American Cancer Society. Cancer facts \& figures 2015. Atlanta: American Cancer Society, 2015.

17. Flowers CR, Armitage JO. A decade of progress in lymphoma: advances and continuing challenges. Clin Lymphoma Myeloma Leuk 2010;10:414-23.

18. Huang T, Shenoy PJ, Sinha R, et al. Development of the Lymphoma Enterprise Architecture Database: a caBIG Silver level compliant system. Cancer Inform 2009;8:45-64.

19. Muringampurath-John D, Jaye DL, Flowers CR, et al. Characteristics and outcomes of diffuse large B-cell lymphoma presenting in leukaemic phase. Br J Haematol 2012;158:608-14.

20. Thavendiranathan P, Grant AD, Negishi T, et al. Reproducibility of echocardiographic techniques for sequential assessment of left ventricular ejection fraction and volumes: application to patients undergoing cancer chemotherapy. J Am Coll Cardiol 2013;61:77-84.

21. Gottdiener JS, Livengood SV, Meyer PS, et al. Should echocardiography be performed to assess effects of antihypertensive therapy? Test-retest reliability of echocardiography for measurement of left ventricular mass and function. J Am Coll Cardiol 1995;25:424-30.

22. Bird BR, Swain SM. Cardiac toxicity in breast cancer survivors: review of potential cardiac problems. Clin Cancer Res 2008;14:14-24.

23. Hunt SA, Baker DW, Chin MH, et al., American College of Cardiology/American Heart Association Task Force on Practice Guidelines (Committee to Revise the 1995 Guidelines for the Evaluation and Management of Heart Failure); International Society for Heart and Lung Transplantation; Heart Failure Society of America. ACC/AHA guidelines for the evaluation and management of chronic heart failure in the adult: executive summary a report of the American College of Cardiology/American Heart Association Task Force on Practice Guidelines (committee to revise the 1995 guidelines for the evaluation and management of heart failure). Circulation 2001;104:2996-3007.

24. Elliott P. Pathogenesis of cardiotoxicity induced by anthracyclines. Semin Oncol 2006;33(3 Suppl 8):S2-7.

25. Schwartz RG, McKenzie WB, Alexander J, et al. Congestive heart failure and left ventricular dysfunction complicating doxorubicin therapy. Seven-year experience using serial radionuclide angiocardiography. Am J Med 1987;82:1109-18.

26. Cardinale D, Colombo A, Bacchiani G, et al. Early detection of anthracycline cardiotoxicity and improvement with heart failure therapy. Circulation 2015;131:1981-8.

27. Lenihan DJ, Oliva S, Chow EJ, et al. Cardiac toxicity in cancer survivors. Cancer 2013:119(Suppl 11):2131-42.

28. Lenihan DJ, Cardinale D, Cipolla CM. The compelling need for a cardiology and oncology partnership and the birth of the International CardiOncology Society. Prog Cardiovasc Dis 2010;53:88-93. 